\title{
Quasi-Kernels for Oriented Paths and Cycles
}

\author{
Stephen Bowser, Charles Cable \\ Department of Mathematics, Allegheny College, Meadville, USA \\ Email: sbowser@allegheny.edu
}

Received February 2, 2012; revised March 2, 2012; accepted March 21, 2012

\begin{abstract}
If $D$ is a digraph, then $K \subseteq V(D)$ is a quasi-kernel of $D$ if $D[K]$ is discrete and for each $y \in V(D)-K$ there is $x \in K$ such that the directed distance from $y$ to $x$ is less than three. We give formulae for the number of quasi-kernels and for the number of minimal quasi-kernels of oriented paths and cycles.
\end{abstract}

Keywords: Digraph; Quasi-Kernel; Path; Cycle

\section{Introduction}

Notation For a digraph $D, V(D)$ and $A(D)$ denote its vertex set and arc set, respectively. If $U \subseteq V(D)$, then $D[U]$ denotes the subdigraph of $D$ induced by $U$ and in $(U)$ and out $(U)$ denote, respectively, the inand out-neighborhoods of $U$. If $U=\{x\}$, these latter sets may be written in $(x)$ and out $(x)$.

Definition 1 A quasi-kernel $K$ of a digraph $D$ is a subset of $V(D)$ satisfying two properties:

1) The quasi-absorbing property: $\forall y \in V(D)-K$, $\exists x \in K$ such that the directed distance in $D$ from $y$ to $x$ is either one or two;

2) Independence: there is no arc in $A(D)$ between vertices of $K$.

If $K$ is a quasi-kernel for digraph $D, x \in K$ and $y \in V(D)-K$ and the directed distance from $y$ to $x$ in $D$ is either one or two, then we will say that $x$ quasi-absorbs $y$.

By [1], every digraph has at least one quasi-kernel. The number of quasi-kernels possessed by various classes of digraphs has been addressed in several papers. For example, in [2] Gutin et al. give necessary and sufficient conditions for a digraph to have at least three quasi-kernels. Clearly, any independent superset of a quasi-kernel is a quasi-kernel. In [3] we give sufficient conditions for a digraph to have at least three minimal quasi-kernels. In [4], Heard and Huang provide sufficient conditions for at least three disjoint quasi-kernels in certain classes of digraphs. In the present work, we address the problem of counting the number of quasi-kernels and the number of minimal quasi-kernels of oriented paths and cycles.

Notation For a digraph $D, k(D)$ denotes the number of distinct quasi-kernels of $D$ and $m(D)$ denotes the number of minimal quasi-kernels of $D$.
Our approach is to recursively construct all quasikernels and all minimal quasi-kernels of various classes of digraphs in order to count them. The following general lemma will be useful for the examination of these special classes.

Lemma 1 If $D_{1}$ and $D_{2}$ are digraphs such that $V\left(D_{1}\right) \cap V\left(D_{2}\right)=\{x\}$, where $x$ is a sink in both $D_{1}$ and $D_{2}$, then $k\left(D_{1} \cup D_{2}\right)=k\left(D_{1}\right) k\left(D_{2}\right)$ and $m\left(D_{1} \cup D_{2}\right)=m\left(D_{1}\right) m\left(D_{2}\right)$.

Proof. Using the notation from the statement of the lemma, if $K_{i}$ is a quasi-kernel for $D_{i}$ for $i=1,2$, then $K=K_{1} \cup K_{2}$ clearly satisfies the quasi-absorbing property for $D=D_{1} \cup D_{2}$ and $D$ has no arcs between a vertex of $K_{1}-\{x\}$ and one of $K_{2}-\{x\}$, so $K$ inherits independence from $K_{1}$ and $K_{2}$. It follows that $k(D) \geq k\left(D_{1}\right) k\left(D_{2}\right)$.

If on the other hand, $K$ is a quasi-kernel of $D=D_{1} \cup D_{2}$ and $K_{i}=K \cap V\left(D_{i}\right)$ for $i=1,2$, then clearly both $K_{1}$ and $K_{2}$ are independent. Since $x$ is a sink in $D$, a directed path in $D$ lies completely in $D_{1}$ or $D_{2}$. Thus the quasiabsorbing property is satisfied by $K_{1}$ in $D_{1}$ and by $K_{2}$ in $D_{2}$. It follows that every quasi-kernel of $D$ arises as the union of a quasi-kernel of $D_{1}$ and one of $D_{2}$, so $k(D) \leq k\left(D_{1}\right) k\left(D_{2}\right)$. It is clear that $K_{1}$ and $K_{2}$ are both minimal for $D_{1}$ and $D_{2}$ respectively iff $K_{1} \cup K_{2}$ is minimal for $D_{1} \cup D_{2}$, so $m\left(D_{1} \cup D_{2}\right)=m\left(D_{1}\right) m\left(D_{2}\right)$.

\section{Paths}

By Lemma 1, to count the number of quasi-kernels in an arbitrary oriented path, it suffices to consider those with no internal sinks, i.e. the digraphs $P_{n}$ and $P_{m, n}$ defined below.

\section{Notation}

1) $P_{n}$ denotes the directed path on vertices $v_{1}, v_{2}, \cdots, v_{n}$ 
with arc set $\left\{v_{1} \rightarrow v_{2}, v_{2} \rightarrow v_{3}, \cdots, v_{n-1} \rightarrow v_{n}\right\}$.

2) For $m, n \geq 2, P_{m, n}$ denotes the result of identifying the source vertices of $P_{m}$ and $P_{n}$. We will denote the vertices $v_{m}, \cdots, v_{2}, s, u_{2}, \cdots, u_{n}$ where $s=v_{1}=u_{1}$, and $v_{i} \rightarrow v_{i+1}$, and $u_{j} \rightarrow u_{j+1}$.

3) $e(n)$ denotes the number of quasi-kernels of $P_{n}$ containing the vertex $v_{1}$.

There are two quasi-kernels of $P_{6}$ containing $v_{1}$, viz. $\left\{v_{1}, v_{3}, v_{6}\right\}$ and $\left\{v_{1}, v_{4}, v_{6}\right\}$, thus $e(6)=2$. The results we obtain will be expressed in terms of the $e$ function, so our first goal is to obtain a closed-form expression for its value. We start with a useful recursion relation.

Lemma 2 If $n \geq 4$, then $e(n)=e(n-2)+e(n-3)$.

Proof. If $K$ is a quasi-kernel of $P_{n}$ containing $v_{1}$, then, by the quasi-absorbing property, $K$ must contain either $v_{3}$ or $v_{4}$ and, by independence, $K$ cannot contain both, nor can it contain the vertex $v_{2}$. The equation follows.

For some of what follows, it is convenient to extend the domain of the function $e$ using the above recursion relation backwards. Thus, since $e(1)=e(3)=1$ and $e(2)=0$, we set $e(0)=e(-1)=0$ and the recursion relation as stated in Lemma 2 holds for $n \geq 2$.

It is straightforward to confirm that the characteristic equation of the above recursion relation, $r^{3}-r-1=0$, has the following roots:

$$
r_{1}=\alpha+\beta, r_{2}=-\frac{(\alpha+\beta)}{2}+i \sqrt{3} \frac{(\beta-\alpha)}{2} \text {, and } r_{3}=\bar{r}_{2}
$$

where

$$
\alpha=\frac{1}{\sqrt{3}}\left(\frac{\sqrt{27}-\sqrt{23}}{2}\right)^{1 / 3} \text { and } \beta=\frac{1}{\sqrt{3}}\left(\frac{\sqrt{27}+\sqrt{23}}{2}\right)^{1 / 3} \text {. }
$$

The solution of the recursion relation can be written $e(n)=x_{1}\left(r_{1}\right)^{n}+x_{2}\left(r_{2}\right)^{n}+x_{3}\left(r_{3}\right)^{n}$, where $x_{1}, x_{2}$ and $x_{3}$ are selected to satisfy the initial conditions $e(0)=e(2)=0$ and $e(1)=1$. The straightforward solution of the $3 \times 3$ system provides the following solution. (The identity $\alpha \beta=1 / 3$ was used in obtaining these expressions.)

$$
x_{1}=\frac{r_{1}^{2}}{2 r_{1}+3}, \overline{x_{3}}=x_{2}=\frac{1}{2\left(2 r_{1}+3\right)}\left(-r_{1}^{2}+\frac{r_{1}+3}{\sqrt{3}(\alpha-\beta)} i\right) \text {. }
$$

Theorem 1 For $n \geq 3, e(n)=\left\lfloor\frac{r_{1}^{n+2}}{2 r_{1}+3}+\frac{1}{2}\right\rfloor$

Proof. Notice that

$$
\frac{1}{\sqrt{3}}<\beta<\frac{1}{\sqrt{3}}\left(\frac{2 \sqrt{27}}{2}\right)^{1 / 3}=1 .
$$

So, since $\alpha \beta=1 / 3$,

$$
\begin{aligned}
& \left|r_{2}\right|=\left|r_{3}\right|=\sqrt{\alpha^{2}+\beta^{2}-\alpha \beta}=\sqrt{\frac{1}{9 \beta^{2}}+\beta^{2}-\frac{1}{3}}<\sqrt{\frac{1}{3}+1-\frac{1}{3}} \\
& =1 .
\end{aligned}
$$

It can also be shown that $\left|x_{2} r_{2}^{3}\right|=\left|x_{3} r_{3}^{3}\right|<1 / 4$. (We omit the details, but this follows in a straightforward way, making use of the facts that

$$
3(\alpha-\beta)^{2}=3\left((\alpha+\beta)^{2}-4 \alpha \beta\right)=3 r_{1}^{2}-4
$$

and that $r_{1}^{3}-r_{1}-1=0$. Thus, for all $n \geq 3$,

$$
\left|e(n)-x_{1} r_{1}^{n}\right| \leq\left|x_{2} r_{2}^{n}\right|+\left|x_{3} r_{3}^{n}\right|<1 / 2 .
$$

Since $e(n)$ is integer valued, the result follows.

Theorem 2 For all integers $n \geq 1, k\left(P_{n}\right)=e(n+3)$ and $m\left(P_{n}\right)=e(n+2)$.

Proof. By the quasi-absorbing property, the first vertex of a quasi-kernel of $P_{n}$ for $n \geq 3$ must be $v_{1}, v_{2}$, or $v_{3}$, thus $k\left(P_{n}\right)=e(n)+e(n-1)+e(n-2)$. Using Lemma 2 twice produces the desired equation. The first two values of $n$ can be considered directly:

$$
k\left(P_{1}\right)=k\left(P_{2}\right)=1=e(4)=e(5) .
$$

The only quasi-kernels of $P_{n}$ which are not minimal are those that contain both $v_{1}$ and $v_{3}$. There are clearly $e(n-2)$ of these, so $m\left(P_{n}\right)=e(n)+e(n-1)$. Again, the recursion relation produces the desired result.

Theorem 3 For all integers $m, n \geq 2$,

$$
\begin{aligned}
k\left(P_{m, n}\right)= & e(m) \cdot e(n)+e(m+1) \cdot e(n-3) \\
& +e(m+2) \cdot e(n+1)
\end{aligned}
$$

Proof. As noted in its definition, the digraph $P_{m, n}$ contains subdigraphs isomorphic to $P_{m}$ and $P_{n}$. We will abuse the notation by using $P_{m}$ and $P_{n}$ to denote subgraphs induced by $\left\{s, v_{2}, \cdots, v_{m}\right\}$ and $\left\{s, u_{2}, \cdots, u_{n}\right\}$, respectively. The set of all quasi-kernels of $P_{m, n}$ can be partitioned according to how the quasi-absorbing property is satisfied for the vertex $s$. Let $K$ be a quasi-kernel of $P_{m, n}$ and consider cases.

1) $K$ contains $s$.

In this case $K \cap V\left(P_{m}\right)$ is a quasi-kernel of $P_{m}$ containing $s=v_{1}$ and $K \cap V\left(P_{n}\right)$ is a quasi-kernel of $P_{n}$ containing $s=u_{1}$. Thus, this case contributes $e(m) e(n)$ quasi-kernels of $P_{m, n}$.

2) $K$ contains $v_{2}$ or $v_{3}$, but not $s, u_{2}$, nor $u_{3}$.

Note that the quasi-absorbing property requires that $u_{4} \in K$. In this case $K \cap V\left(P_{m}\right)$ is one of the $e(m-1)+e(m-2)$ quasi-kernels of $P_{m}$ with first vertex either $v_{2}$ or $v_{3}$ and $K \cap V\left(P_{n}\right)$ is one of the $e(n-3)$ quasi-kernes of the subgraph of $P_{m, n}$ induced by $\left\{u_{4}, \cdots, u_{n}\right\}$ containing $u_{4}$. There are

$$
(e(m-1)+e(m-2)) e(n-3)=e(m+1) e(n-3)
$$

such sets $K$.

3) $K$ contains $u_{2}$ or $u_{3}$, but not $s=v_{1}, v_{2}$ nor $v_{3}$.

This is the preceding case with $m$ and $n$ reversed. It contributes $e(m-3) e(n+1)$ additional quasi-kernels. 
4) $K$ contains one vertex from $\left\{v_{2}, v_{3}\right\}$ and one from $\left\{u_{2}, u_{3}\right\}$ but does not contain $s$.

Here, $K \cap V\left(P_{m}\right)$ is one of the $e(m-2)+e(m-1)$ quasi-kernels of $P_{m}$ not containing $S=v_{1}$ and $K \cap V\left(P_{n}\right)$ is a quasi-kernel of $P_{n}$ not containing $s=u_{1}$, of which there are $e(n-2)+e(n-1)$. Thus we have a final

$$
\begin{aligned}
& (e(m-2)+e(m-1))(e(n-2)+e(n-1)) \\
= & e(m+1) e(n+1)
\end{aligned}
$$

quasi-kernels.

Summing the contributions over this mutually exclusive and exhaustive list of cases:

$$
\begin{aligned}
k\left(P_{m, n}\right)= & e(m) e(n)+e(m+1) e(n-3) \\
& +e(m-3) e(n+1)+e(m+1) e(n+1)
\end{aligned}
$$

The last two terms can be combined using Lemma 2 since

$$
\begin{aligned}
& e(m-3)+e(m+1) \\
= & e(m-3)+e(m-2)+e(m-1) \\
= & e(m)+e(m-1)=e(m+2) .
\end{aligned}
$$

Theorem 4 For all integers $m, n \geq 2$,

$$
m\left(P_{m, n}\right)=2 e(m-3) \cdot e(n-3)+e(m+1) \cdot e(n+1)
$$

Proof. Throughout this proof we follow the notation introduced on page 2. Note first that any quasi-kernel of $P_{m, n}$ containing the source (s) and also either $v_{3}$ or $u_{3}$ is not minimal. On the other hand, any quasi-kernel containing the source but contains neither $v_{3}$ nor $u_{3}$ must contain both $v_{4}$ and $u_{4}$. It is easy to see that such a quasikernel is minimal. Thus there are $e(m-3) \cdot e(n-3)$ minimal quasi-kernels containing the source.

The table in Figure 1 depicts the relevant fragments of all possible quasi-kernels not containing the source. The possiblilities are sorted according to the subscripts on the $v$ 's and secondarily to the subscript on the $u$ 's. The leftmost column enumerates the cases for reference herein. The right-most column gives, in the case of a non-minimal quasi-kernel, an example of an extraneous vertex and, otherwise, the number of minimal quasi-kernels containing the given fragment. For example in case $1, v_{2}$ could be removed from a quasi-kernel containing that fragment and leave a set which is still a quasi-kernel whereas in case 8 , the given fragment could be extended by any of the $e(m-4)$ quasi-kernels of $P_{m, n}\left[\left\{v_{5}, \cdots, v_{m}\right\}\right]$ containing $v_{5}$ and, independently, any of the $e(n-2)$ quasi-kernels of $P_{m, n}\left[\left\{u_{3}, \cdots, u_{n}\right\}\right]$ containing $u_{3}$ to yield a minimal quasi-kernel of $P_{m, n}$. Certain of the vertices have been left off the table if their presence in the minimal quasi-kernel is neither mandatory nor forbidden in the case depicted. For example in row $7, u_{5}$ is not depicted since, in the configuration shown, it is present in some minimal quasi-kernels and absent in others.

Lemma 2 can be used to simplify the sum of the terms in the right column which contribute to $m\left(P_{m, n}\right)$. Thus, rows 6-8 sum to $e(m-4) \cdot e(n+1)$, rows $10-12$ sum to $e(m-2) \cdot e(n-2)$, and rows $13-14$ sum to $e(m-3) \cdot e(n+1)$. The last two of these expressions sum to $e(m) \cdot e(n+1)$, and thus we have the number of minimal quasi-kernels of $P_{m, n}$ which do not contain the source:

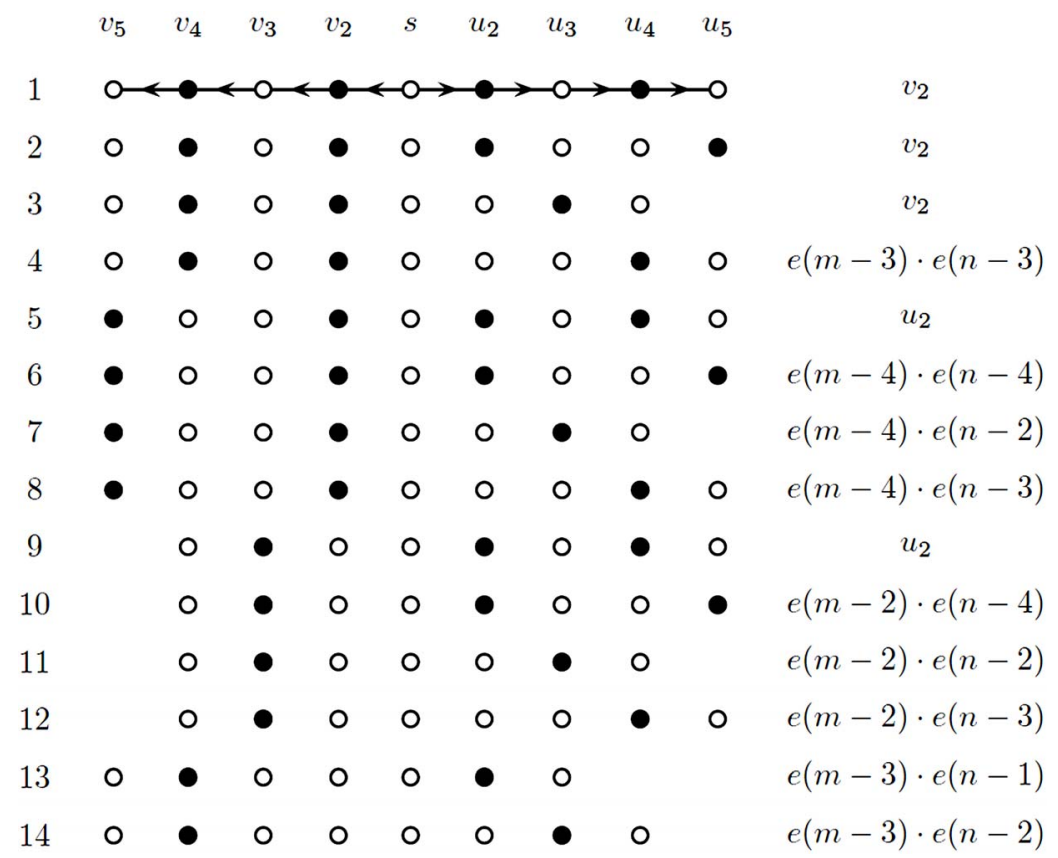

Figure 1. $P_{m, n}$. 


$$
\begin{aligned}
& e(m-3) \cdot e(n-3)+[e(m-4)+e(m)] \cdot e(n+1) \\
= & e(m-3) \cdot e(n-3)+e(m+1) \cdot e(n+1) .
\end{aligned}
$$

Combining this with the number already obtained for those that contain the source gives the desired result.

As stated at the beginning of this section, Theorems 2, 3 and 4 used with Lemma 1 provide the number of quasikernels and the number of minimal quasi-kernels for arbitrary oriented paths.

\section{Cycles}

Counting quasi-kernels for oriented cycles can almost be reduced to counting those of oriented paths. Of course any oriented cycle can be obtained by identifying the "leaf" vertices of an oriented path. The reverse process we shall call "splitting".

Theorem 5 If $x$ is a sink of a oriented cycle $C$ and $P$ is the oriented path obtained by splitting $C$ at $x$, then $k(C)=k(P)$ and $m(C)=m(P)$.

Proof. Let the two vertices of $P$ whose identification produce $x$ in $C$ be $x^{\prime}$ and $x^{\prime \prime}$. Every quasi-kernel of a digraph must contain all the sinks of that digraph, so $K$ is a quasi-kernel of $C$ iff $K^{\prime}=K \cup\left\{x^{\prime}, x^{\prime \prime}\right\}-x$ is a quasikernel of $P$. In particular, $K$ is minimal for $C$ iff $K^{\prime}$ is minimal for $P$. The result follows.

Theorem 6 If $C$ is a oriented cycle of order $n$ with no sink, then $m(C)=k(C)=2 e(n+1)+e(n-2)$.

Proof. Observe first that every quasi-kernel of such a $C$ is minimal, so $m(C)=k(C)$. $C$ is the result of identifying vertices $v_{1}$ and $v_{n+1}$ of $P_{n+1}$. Use the vertex names from $P_{n+1}$ ( $v_{1}$ for the identified vertices). If $n \geq 4$ and $K$ is a quasi-kernel for $C$, then there are three possibilities.

1) $K$ contains $v_{1}$.

These quasi-kernels are exactly the images under the identification above of a quasi-kernel of $P_{n+1}$ containing $v_{1}$. There are $e(n+1)$ of these.

2) $K$ contains $v_{2}$.

Again, there are $e(n+1)$ such quasi-kernels.

3) $K$ contains $v_{3}$ but not $v_{1}$.

Since $K$ contains neither $v_{1}$ nor $v_{2}$, it must contain $v_{n}$. There are therefore $e(n-2)$ such quasi-kernels.

The quasi-absorbing property requires that $K$ contain at least one of the vertices $v_{1}, v_{2}$ and $v_{3}$. On the other hand, independence ensures that the cases above are mutually exclusive.

\section{REFERENCES}

[1] V. Chvátal and L. Lovász, "Every Directed Graph Has a Semi-Kernel," Hypergraph Seminar, Lecture Notes in Mathematics, Vol. 441, Springer-Verlag, Berlin, 1974, p. 175.

[2] G. Gutin, K. M. Koh, E. G. Tay and A. Yeo, "On the Number of Quasi-Kernels in Digraphs,” Journal of Graph Theory, Vol. 46, No. 1, 2004, pp. 48-56. doi:10.1002/jgt.10169

[3] S. Bowser and C. Cable, "At Least Three Minimal Quasi-Kernels,” Discrete Applied Mathematics, Vol. 160, No. 4-5, 2012, pp. 673-675. doi:10.1016/j.dam.2011.08.017

[4] S. Heard and J. Huang, "Disjoint Quasi-Kernels in Digraphs,” Journal of Graph Theory, Vol. 58, No. 3, 2008, pp. 251-260. doi:10.1002/jgt.20310 